\title{
Development and Evaluation of a Gaze Detection System for Walking and Texting
}

\author{
- Gaze Detection by Determining Facial Angles with Face Recognition Technology, \\ and Gaze Detection through Eye Detection -
}

\author{
Kento SASAO, Kazuki OGI, Jue ZHANG and Noboru SUGAMURA \\ Kogakuin University, 1-24-2 Nishi-Shinjuku, Shinjuku-ku, Tokyo 163-8677, Japan
}

\begin{abstract}
Using a smart phone while walking has been becoming a social issue. Acceleration-based applications to prevent texting while walking are now being developed, but their accuracy rate is low and false detection occurs because only a single element is used based on the required number of steps. The problem in texting while walking is that people are gazing at the smart phone to operate it while walking. Therefore, the ultimate objective of this study was set to develop a highly accurate application in consideration of false detection issues, focusing on the face authentication technology and eye detection with the in-camera. We developed a trial application, conducted eye gaze detection verification experiments using the face authentication technology, and achieved a high accuracy rate of about $96 \%$. In addition, eye detection initially showed a low detection rate in the study, but we were able to minimize false positives by using a cascade classifier. Based on this result, we believe that this technology can be used to detect the gaze of smart phone users.
\end{abstract}

Keywords: Texting while walking, Facial recognition, Application development

\section{INTRODUCTION}

As the use of smartphones becomes more widespread, a social problem is emerging across the globe, wherein smartphone users walk and text at the same time, and cause accidents due to distraction. Various preventive measures have been designed and developed inside and outside Japan to address this issue [1, 4, 10, 12, 13, 16, 17]. In Japan, between 2010 and 2013, there were 122 accidents caused by pedestrians using their phones; in 92 of these, those responsible for the accidents were distracted because they were looking at their phone screens, answering a phone call, or otherwise using their phones [7,14]. NTT DoCoMo, a Japanese wireless carrier, released a video in 2014 showing a computer simulation of what the Shibuya crossing would look like if 1,500 people trying to cross the street were texting while walking, and the video garnered much attention [9]. In response to this social problem, Japanese authorities have posted signs in public places to alert people to the dangers of using smartphones while walking. Also, numerous applications have been developed which exploit smartphones' built-in sensors and camera to prevent the use of phones while walking, and these are available to the public. Most existing applications and studies on these applications, however, primarily rely on a single sensor, the accelerometer, to detect steps and examine method accuracy. Also, there has been insufficient discussion of how to detect texting while walking, under conditions that tend to produce false positives, and how to minimize false positives. In an experiment using the smartphone's front camera, subjects walked and texted with the phone's front view displayed on their screen, so that they would be aware of what was ahead of them. However, this method was reported to be ineffective as a user's gaze is fixed on specific points on the screen when they walk and text, and their field of vision is typically limited to only $1 / 20$ of normal [9]. In sum, there have been no proven solutions to the problems associated with users visually focusing on their smartphone while walking, which are the core of the 'walking and texting' problem, or to the problem of false positives that occur in certain scenarios and usermovement conditions. This study focuses on one of the most central issues involved in texting while walking: users' focused gaze on the phone screen during smartphone use; and examines the availability and applications of technology that determines whether users are looking at their screen, by detecting facial angles in real time. In addition, in order to overcome limitations in the above technique due to potential insensitivity in the built-in camera, and problems caused by background imagery captured by the camera, this study explores the possibility of using eye detection to determine whether a person is gazing at their phone, and examines new methods to detect gaze direction. 


\section{SENSITIVITY TO DANGER}

In recent years, an increasing number of people are becoming insensitive to 'danger'; and this has led to extensive discussion of how to engender in people a healthy sense of danger, and conversely, of safety. In this discussion, experts have raised concerns that many in today's society are so used to being safe and secure that they are growing insensitive to potential sources of danger; and note that many consider safety as something that is simply provided, and thus are unwilling to protect their own safety. In addition, some lack the common sense and experience that are expected to have been cultivated over time, and this makes them likely to engage in dangerous activities that may impact others; one example of which is texting while walking.

When we examined cases in which people used their smartphones while walking, most usage was found to be in relatively innocuous situations, where the tasks were neither so urgent that users were required to continue working on them while walking, nor so difficult that they had to pause and focus. Also, by nature, walking only requires use of the legs, leaving the brain and hands free for other activities. Thus, the time spent walking may be seen as 'free time', until one's destination is reached. In addition, the rapidly increasing portability and convenience of modern communication systems has enabled multi-tasking, which encourages the mindset that when we have time to spare, we should be using our brain and hands, which are otherwise 'not in use'. Sensitivity to danger is founded on one's personal experience, as well as on information from the external world. To address an insufficient sense of danger, then, it is often necessary to enhance sensitivity through external stimuli.

This paper examines the effectiveness of using technology to enhance smartphone-user sensitivity to danger (as well as safety), and thus ultimately create a safer user experience.

\section{VERIFICATION EXPERIMENT 1 ON DETEC- TIONANGLE IN THE FACE AUTHENTICATION TECHNOLOGY}

\subsection{Developing the facial recognition application}

To determine whether a person is gazing at the screen while using his or her smartphone, it is first necessary to determine the range in which the smartphone screen is within the user's visual field. This can be done by using the phone's built-in camera for face recognition and determining the facial angles in real time. To this end, a number of studies are being conducted, and multiple applications are being developed for both Android and iOS operating systems. Though there are numerous applications for Android, problems still persist; and only two such applications have been developed for iOS. Given this, and the fact that iOS smartphones have a roughly 50\% market share in Japan, matching that of Android, we focused on the iOS system in developing our applications in this study. Facial recognition can be achieved, in this system, using methods involving SquareCam, OpenCV, CIDetector, and/or feature extraction, for example. However, beyond a few investigations limited to specific functions, there have been no studies on these methods, and no verification tests regarding their accuracy in facial detection. Therefore, we developed applications employing each of these four methods (SquareCam, OpenCV, CIDetector, and feature extraction), and conducted experiments to test their accuracy. The results showed that Apple's SquareCam library showed the greatest accuracy in facial detection $[6,8]$. It is reported that up to 20 degrees from the reference position where the full front face can be viewed straight in front can be recognizable; however, if the face is partly covered with sunglasses or a mask, the face can be hardly recognized. Experiment 1 investigated differences between two groups: the range of face recognition (angle of the face) with the developed application, and the actual field of vision of the user operating a smart phone (angle of the face).

\subsection{Methodology}

The experiment was designed to analyze two situations: (1) The frontal face position is used as the reference $\left(0^{\circ}\right)$, and up to how much degree of the face the application is capable of recognizing it is determined when the person changes the direction of the face to right, left, up and down, and (2) the viewing angle of the person is determined by examining at what degree of the face to right, left, up and down the iPhone goes out of the sight of the person. To determine the range of viewing angle, the person was asked to tell when the image on the iPhone screen could no longer be identified except for its colors or the image began to blur, and photographs were taken from the top or left of the person. A total of 5 subjects ( 3 men and 2 women) aged 21 or 22 participated in the experiment. Figure 1 shows the flow of the experiment. For each subject, 8 sets a day ( 3 times per set), 4 for right and left, and another 4 for up and down, were conducted for 5 days, meaning that a total of 120 trial (60 trials each for right and left, and for up and down) were conducted per one subject. Since there were 5 subjects, a total of 600 

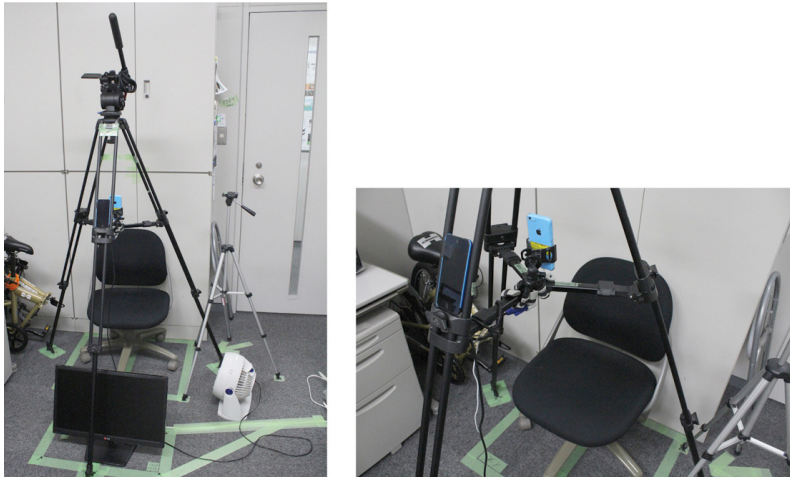

Figure 1: Settings of verification experiment 1

trials were performed. The distance between the face and the camera was set to $27 \mathrm{~cm}$, the average value obtained from pre-experiment measurement. The distance between the floor and the iPhone was set in such a way that the in-camera was on the same horizontal line with the height of the eye of the subject (Figure 2).

[Additional experiment] After the experiment explained above was completed, an additional face recognition experiment was conducted for the same 5 subjects. This time, they wore sunglasses or a mask. A total of 20 trials, 4 trials for each of the 5 subjects ( 2 with sunglasses, and another 2 with a mask) were conducted.

\subsection{Analysis}

(1) For the angle of the face directed to left, the midpoint of the face was determined by drawing a line between the right and left end of the face (width of the face), and between the nose and the center of the back of the head; (2) the angle of the face is measured by drawing a line from the midpoint determined in (1) to the tip of the nose; and (3) the same procedure was taken for the angle of the face directed to right. (4) For the upward angle, the midpoint of the face was determined by drawing a line between the tip of the nose and the center of the back of the head, and between the chin and the top of the head. Two points were also determined, each on the chin and the tip of the nose; (5) when the subject looked upward, two points were again determined, each on the chin and the tip of the nose, and lines were drawn between these points. The change in angle based on the reference lines were determined, and (6) the same procedure was taken for the downward angle of the face.

The red lines represent auxiliary lines to indicate the positions of the iPhone and the in-camera, while the yellow lines are those used to determine the range and midpoints of the face (Figure 3).
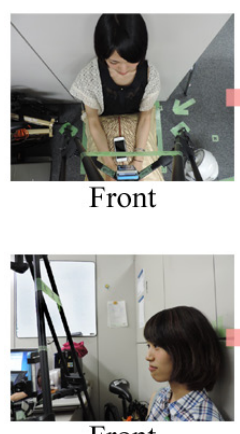

Front

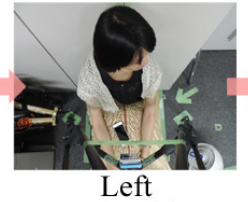

(Facia tra

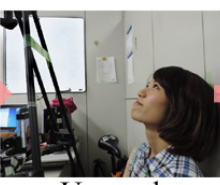

Upward (Facial recognition range)

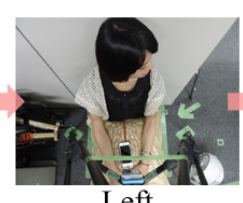

Left

(Field of view range)
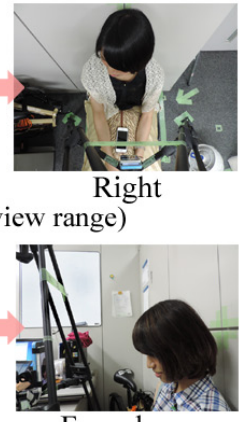

Face down

(Field of view range)

Figure 2: Verification experiment 1

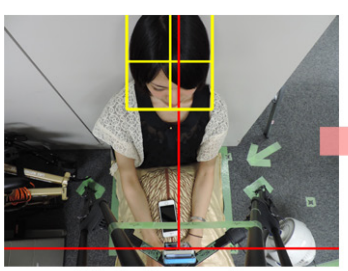

(1) Reference line

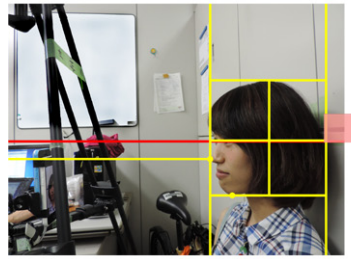

(4) Reference line

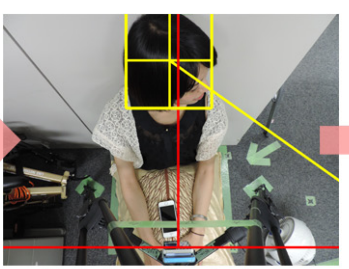

(2) Left angle calculation

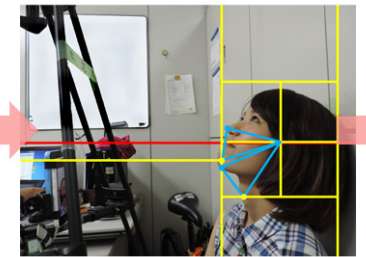

(5) Upward angle calculation

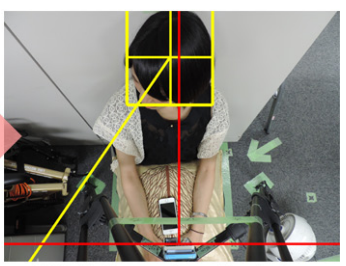

(3) Right angle calculation

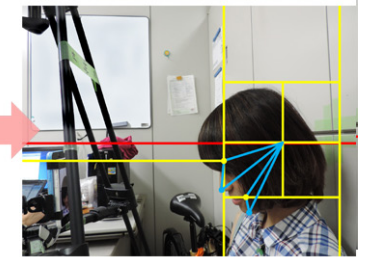

(6) Face down angle calculation

Figure 3: Auxiliary lines to measure the angle 


\subsection{Results and consideration}

The verification experiment was conducted with the detection application, and a t-test was carried out to compare the averages between the range of face recognition and that of the field of vision. As a result, no significant difference was observed among $75 \%$ of the total (left side of Table 1), indicating that the developed detection application can be possibly used because the difference in angle between the ranges of face recognition and field of vision were small. But since No. 1 and 4 in Table 1 (left) suggest significant differences among individuals, differences among individual subjects were separately calculated, resulting in a significant difference of $65 \%$ (31 out of 48) (right side of Table 1). These differences must have been caused by differences in the length of hair and the size of the face. Therefore, further control is required within the system.

Table 1: t-test on face recognition range and viewing angle (Top) and on differences among individuals (Bottom)

\begin{tabular}{|c|c|c|c|c|c|c|c|c|c|}
\hline \multicolumn{10}{|c|}{ Test of Facial recognition \& Field of view range (p-value) } \\
\hline & & \multicolumn{2}{|c|}{$\mathrm{L}$} & \multicolumn{2}{|c|}{$\mathrm{R}$} & \multicolumn{2}{|c|}{$\mathrm{U}$} & \multicolumn{2}{|c|}{$\mathrm{D}$} \\
\hline \multicolumn{2}{|c|}{1} & \multicolumn{2}{|c|}{0.045} & \multicolumn{2}{|c|}{0.087} & \multicolumn{2}{|c|}{0.060} & \multicolumn{2}{|c|}{0.007} \\
\hline \multicolumn{2}{|c|}{2} & \multicolumn{2}{|c|}{0.672} & \multicolumn{2}{|c|}{0.407} & \multicolumn{2}{|c|}{0.153} & \multicolumn{2}{|c|}{0.474} \\
\hline \multicolumn{2}{|c|}{3} & \multicolumn{2}{|c|}{0.534} & \multicolumn{2}{|c|}{0.869} & \multicolumn{2}{|c|}{0.149} & \multicolumn{2}{|c|}{0.145} \\
\hline \multicolumn{2}{|c|}{4} & \multicolumn{2}{|c|}{0.004} & \multicolumn{2}{|c|}{0.007} & \multicolumn{2}{|c|}{0.117} & \multicolumn{2}{|c|}{0.097} \\
\hline \multicolumn{2}{|c|}{5} & \multicolumn{2}{|c|}{0.710} & \multicolumn{2}{|c|}{0.422} & \multicolumn{2}{|c|}{0.042} & \multicolumn{2}{|c|}{0.054} \\
\hline \multicolumn{10}{|c|}{ Differences among individuals test (p-value) } \\
\hline & & L1 & L2 & $\mathrm{R} 1$ & $\mathrm{R} 2$ & U1 & U2 & D1 & D2 \\
\hline 1 & \multirow{3}{*}{4} & 0.000 & 0.001 & 0.036 & 0.014 & 0.000 & 0.000 & 0.089 & 0.001 \\
\hline 2 & & 0.900 & 0.006 & 0.001 & 0.000 & 0.000 & 0.000 & 0.301 & 0.114 \\
\hline 3 & & 0.000 & 0.003 & 0.104 & 0.930 & 0.063 & 0.351 & 0.377 & 0.926 \\
\hline 1 & \multirow{3}{*}{5} & 0.000 & 0.000 & 0.009 & 0.007 & 0.004 & 0.005 & 0.002 & 0.000 \\
\hline 2 & & 0.921 & 0.924 & 0.249 & 0.713 & 0.004 & 0.026 & 0.038 & 0.678 \\
\hline 3 & & 0.001 & 0.000 & 0.012 & 0.000 & 0.051 & 0.013 & 0.044 & \begin{tabular}{|l|l|}
0.123 \\
\end{tabular} \\
\hline
\end{tabular}

* L: Left, R:Right, U: Upward, D: Face down,

L1-R1-U1-D1: facial recognition range,

L2-R2-U2-D2: field of view range
[Results of the additional experiment] 10 trials were conducted with sunglasses and the face was recognized $100 \%$ because the face characteristics other than the eyes and the facial contour were clearly visible even with sunglasses. But for trials with a mask, the half of the face was covered with the mask, which made it difficult to detect the facial characteristics and the facial contour, resulting in only 2 successes out of 10 .

\section{VERIFICATION EXPERIMENT 2 ON DETEC- TIONANGLE INTHE FACEAUTHENTICATION TECHNOLOGY}

\subsection{Objectives and overview}

Based on the resulting individual differences in experiment 1 , we made improvement so that the face recognition mark will be automatically adjusted depending on the face size of the subject (Figure 4). For the same 5 subjects in experiment 1, the upward angle limit of recognition limit and angle of the vision field were measured with the fixed smart phone, on the assumption that the subjects were operating it (Figure 5).

Since the results of experiment 1 showed that the up and down angle was smaller than the right and left angle, and at normal times people mostly check their forward situation or look at the smart phone screen, only upward angle experiment was conducted. The same methods, environment, and analytical methods were used with those for experiment 1 .

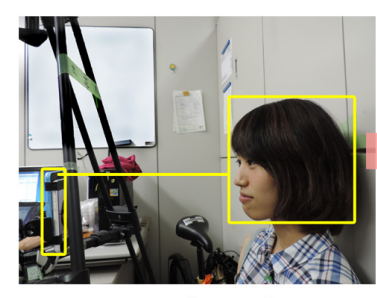

Experiment 1

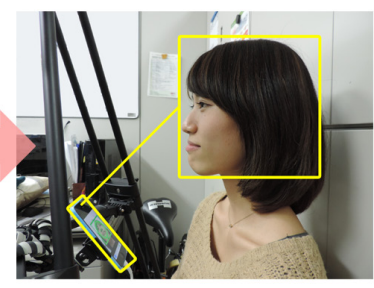

Experiment 2
Figure 5: Smart phone angle difference between experiments 1 and 2

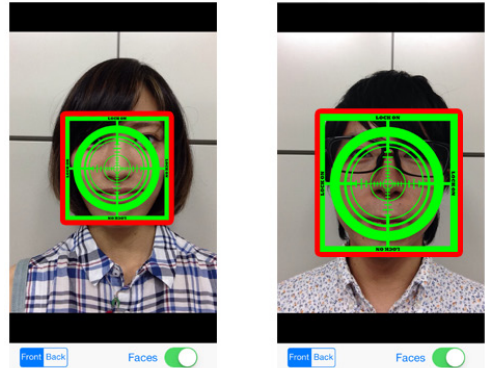

Screen Display [revised]

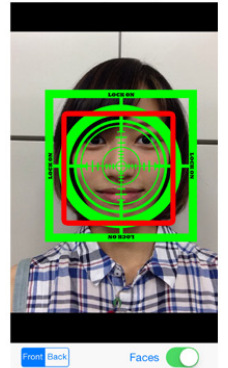

Screen Display [before correction]

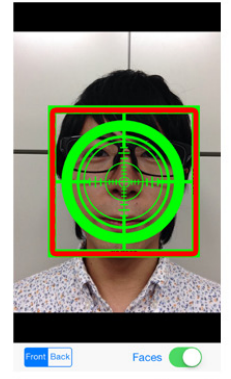

(red: processing by the iPhone; green: recognition mark)

Figure 4: Improvement in the face recognition frame indication 
Table 2: t-test on differences between experiments 1 and 2

\begin{tabular}{|c|c|c|}
\hline \multicolumn{3}{|c|}{ Comparison with experiment 1 and experiment 2 ( $\mathrm{p}$ value) } \\
\hline $\mathrm{p}<0.05$ & FRL & FL \\
\hline Subject 1 & 0.686 & 0.326 \\
\hline Subject 2 & 0.204 & 0.662 \\
\hline Subject 3 & 0.667 & 0.117 \\
\hline Subject 4 & 0.588 & 0.700 \\
\hline Subject 5 & 0.801 & 0.941 \\
\hline \multicolumn{3}{|c|}{ Yellow Marker: significant difference } \\
\hline
\end{tabular}

*FRL : facial recognition Limit, FL : Field Limit

\subsection{Results and consideration}

The face recognition mark was successfully adjusted. The same experimental environment with experiment 1 was used for experiment 2, expect for the angle of the iPhone. In experiment 1 , the iPhone was placed parallel to the face, while it was placed at the inclination that reproduced the actual use condition in experiment 2. The differences in the accuracy of face recognition between experiments 1 and 2 (Table 2) were small, indicating that the face can be recognized at the actual situation where the person is using a smart phone.

\section{VERIFICATION EXPERIMENT 3 ON DETEC- TIONANGLE INTHE FACEAUTHENTICATION TECHNOLOGY}

\subsection{Objectives and overview}

The results of experiments 1 and 2 clearly identified the limit of the face recognition angles, but since the experiments were conducted with the iPhone in a fixed position, the distance between the phone and the face was also fixed. Therefore, experiment 3 aimed at identify the maximum and minimum face recognition distances between the smart phone and face by measuring limit distances. We conducted two types of experiments, one with the iPhone in a fixed position and the other with the phone held by the hand, and examined differences between the two.

\subsection{Experimental environment and methodology}

A measuring device was manufactured and the minimum and maximum face recognition distances were measured (Figure 6). A total of 50 persons (40 men and 10 women) aged between 21 and 23 years participated.
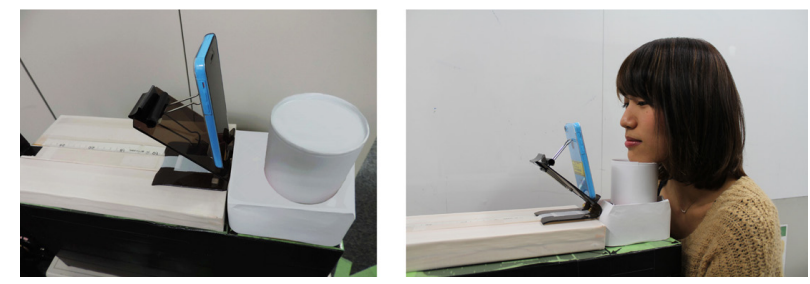

Figure 6: The device and environment of experiment 3
Table 3: t-test on the limit of the face recognition distances

\begin{tabular}{|c|c|c|c|c|}
\hline & Classification & $\begin{array}{l}\text { Measurement } \\
\text { Conditions }\end{array}$ & $\mathrm{CC}$ & F \\
\hline 1 & - & $\begin{array}{l}\text { Minimum Distance - } \\
\text { Height of the face }\end{array}$ & 0.197 & 0.171 \\
\hline 2 & - & $\begin{array}{l}\text { Minimum Distance - } \\
\text { Width of the face }\end{array}$ & 0.327 & 0.020 \\
\hline 3 & - & $\begin{array}{l}\text { Minimum Distance - } \\
\text { Holding }\end{array}$ & 0.542 & 0.000 \\
\hline 4 & $\begin{array}{l}\text { Without } \\
\text { Glasses }\end{array}$ & $\begin{array}{l}\text { Minimum Distance - } \\
\text { Height of the face }\end{array}$ & 0.084 & 0.691 \\
\hline 5 & $\begin{array}{l}\text { Without } \\
\text { Glasses }\end{array}$ & $\begin{array}{l}\text { Minimum Distance - } \\
\text { Width of the face }\end{array}$ & 0.295 & 0.152 \\
\hline 6 & $\begin{array}{l}\text { Without } \\
\text { Glasses }\end{array}$ & $\begin{array}{l}\text { Minimum Distance - } \\
\text { Holding }\end{array}$ & 0.664 & 0.000 \\
\hline 7 & $\begin{array}{l}\text { Wearing } \\
\text { Glasses }\end{array}$ & $\begin{array}{l}\text { Minimum Distance - } \\
\text { Height of the face }\end{array}$ & 0.360 & 0.077 \\
\hline 8 & $\begin{array}{l}\text { Wearing } \\
\text { glasses }\end{array}$ & $\begin{array}{l}\text { Minimum Distance - } \\
\text { Width of the face }\end{array}$ & 0.516 & 0.008 \\
\hline 9 & $\begin{array}{l}\text { Wearing } \\
\text { glasses }\end{array}$ & $\begin{array}{l}\text { Minimum Distance - } \\
\text { Holding }\end{array}$ & 0.592 & 0.002 \\
\hline \multicolumn{5}{|c|}{ Yellow Marker: significant difference } \\
\hline
\end{tabular}

*CC: Correlation Coefficient, F: Significant F

\subsection{Analysis}

Measured data were classified into three groups: data for all the subjects, and data for subjects with and without glasses, and their correlations were analyzed.

\subsection{Results and consideration}

The maximum and minimum distance limits to recognize the face were $2 \mathrm{~m} 50 \mathrm{~cm}$ and $4.5 \mathrm{~cm}$, respectively. Table 3 shows a low correlation factor between the face recognition distance and measurement conditions. The distance limits to recognize the face fall within the range of daily smart phone use conditions, and also the differences between the fixed and hand-held iPhone was small, and not significant. Based on these results, we can say that the face is recognizable under the normal smart phone use conditions and the developed detection application can be used.

\section{VERIFICATION EXPERIMENT 4 ON DETEC- TIONANGLE INTHE FACEAUTHENTICATION TECHNOLOGY}

\subsection{Objectives and overview}

From the results of experiments 1 to 3 , we determined that the "limits of the face recognition angles and the viewing angle of subjects" and the "limits of the face recognition distance" can be utilized to develop an application, which is the objective of this study. The objective of experiment 4 was to check if the determination of the facial angle can be utilized to determine whether or not the smart phone user is gazing by conducting 
experiments under actual walking conditions and to find problems if any. The application this study aims to develop would use the facial angle detection in the face authentication technology, which should be started as a supplement after the determination based on acceleration is completed. Therefore, the verification experiment was also conducted under certain situations where acceleration alone might not be able detect the walking of the smart phone user such as shuffling and short-stepped walking. After the experiment, a questionnaire survey was conducted on changes in consciousness among the subjects toward texting while walking and the evaluation of the detection application used in experiment 4.

\subsection{Methodology}

A total of 25 persons (20 men and 5 women) aged between 21 and 23 years participated in this experiment. They were asked to use a smart phone as usually they do without any specified postures and the way they hold it imposed on them. Areas not susceptible to illuminance and weather variations were selected as experiment routes (Figure 7), and the subjects were asked to wear a head-mount camera to record their gaze, and how many times and when their face was recognized during the experiment was also recorded. Before starting the experiment, the subjects were told to make shorter steps than usual at designated places. The routes include an underground passage, escalator, and stairs, which are normally used in daily life, and 10 check points, where the subjects were asked to take a photo as soon as they find any of them.

\subsection{Analysis}

Behavioral data recorded during the experiment and the $\log$ data of the application were laid out in a chronological order, and the times the face was recognized, and the behavior and gaze of the subjects were analyzed.

\subsection{Results and consideration}

The success rate of face recognition was $95.8 \%$ (SD: 2.461), which indicates that the face can be recognized during normal walking, even when there are individual differences, or when persons make unpredictable actions. We investigated places where the application had malfunctioned (approximately 4\%), and found that illuminance in these places such as lighting might be a cause of malfunction. Then, we examined the range of illuminance in which the face can be recognized by the application and that of the experiment routes. As a result, the range of illuminance in which the face was recognized was 4 to 1114 lux (which is the maximum value along the experiment routes) and at 3 lux or below, the face was not recognized. Meanwhile, the illuminance along the routes was in the range of 44 to 1114 lux, which fell within e-recognizable range. The high-dynamic-range imaging (HDR) that automatically adjusts contrast at the time of start up of the iPhone camera application might be the cause of malfunction. Although we only had the results of Japanese participants in this study, since the face recognition was calculated by the outline and characteristic points (such as participant's nose), the result would not be greatly influenced by the size of the face and the skin color, therefore the method of this study should produce a reliable result for all nationalities $[2,3,5,11,15]$.

\section{VERIFICATION EXPERIMENT 1 ON GAZE DETECTION USING EYE DETECTION}

\subsection{Objectives and overview}

We took a photo of the face of the subjects with the smart phone in-camera, and developed an experimental application to detect circles with OpenCV. We verified whether this application was able to detect the eye (iris) as a circle, and investigated any differences in circle detection when the contrast of the photos was changed.
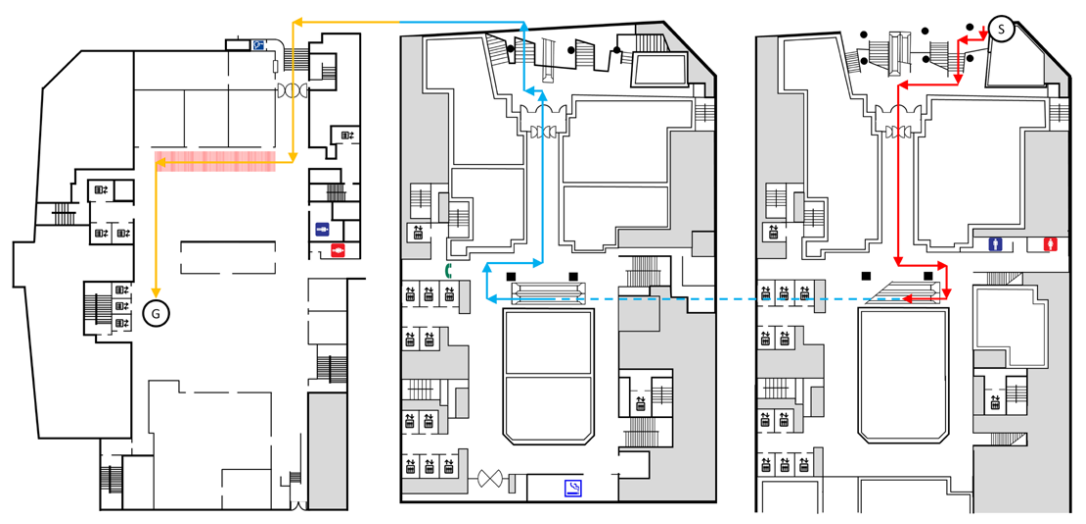

Figure 7: Routes in experiment 4 


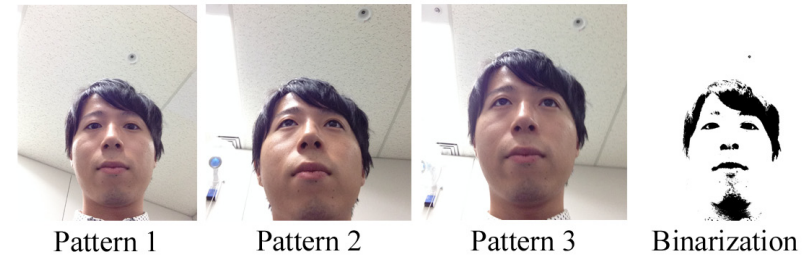

Figure 8: Three facial photo patterns and a binary image

\subsection{Methodology}

A total of 41 college or graduate students (30 men and 11 women) in their 10's to 20' participated in the experiment. We asked them to take photos of their face during usual texting while walking and examined whether or not the developed application was able to detect their eyes. We decided to take photos of the following 3 face patterns, in consideration of differences in vertical facial movement and the case where only eyes moved without moving the face, based on the results of the questionnaire survey that people mostly check their forward situation when they use a smart phone (Figure 8).

- Pattern 1: The face gazing at the smart phone

- Pattern 2: The straight face looking at the front to see the forward situation

- Pattern 3: The downward face with only the eyes looking at the front to see the forward situation

A total of 123 facial photos were collected (41 subjects multiplied by 3 photos without glasses). In addition, in order to examine differences in circle detection rate due to differences in contrast, photos in 8 patterns and binary images using thresholding techniques were created. The 8 patterns are $-80,-60,-40,-20,+20,+40,+60$, and $+80 \%$ against the contrast of the original facial photo as $0 \%$. Thus, a total of 1230 photos (123 photos multiplied by 10 patterns (original photos, those in 8 contrast patterns, and those created by thresholding combined) were used for the circle detection experiment.

\subsection{Results and consideration}

As shown in Figure 9, false detection mainly occurred for photos in contrast different from the original photos. Arcs and non-circle areas were detected as circles. For binary images, circles were not detected. But as shown in Figure 10, the number of circle detections varied depending on the changes in contrast. Figure 11 shows the average number of detections by contrast. The lower the contrast, the less circles were detected, while the higher the contrast, the more false detections occurred. Particularly, the number of false detections on the background tended to be higher. These results show that lower contrast is more likely to reduce false detections.

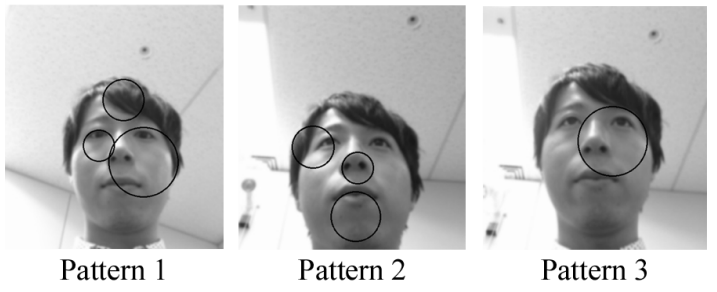

Figure 9: Circle detection result examples

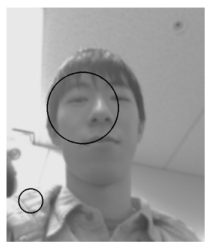

Contrast $-80 \%$

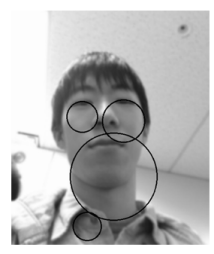

Original image

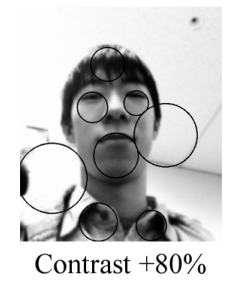

Figure 10: False detection variations due to contrast differences

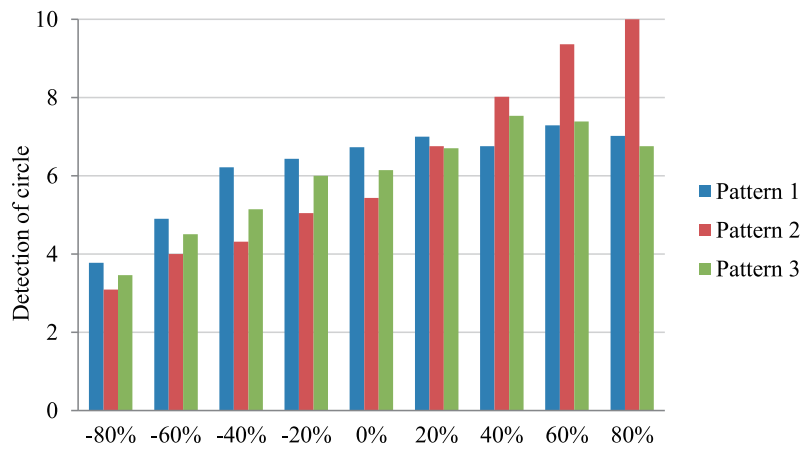

Figure 11: Average number of circle detections by contrast

\subsection{Additional experiment}

For the photos used in experiment 1 , there were many false detections due to the influence of the background, and the eyes were difficult to be detected as circles because they were small in the photos. Therefore, we cut out and enlarged the eye part to see if the eyes were detected or not as shown in Figure 12. As a result, either nothing was detected, or the area along the eyelid was detected as shown in Figure 13.

\subsection{Consideration}

The results of experiment 1 and the additional experiment indicate that the detection of the iris, which is a circle, is very difficult with circle detection, probably because of the accuracy of the camera and because the eyes were narrowed.

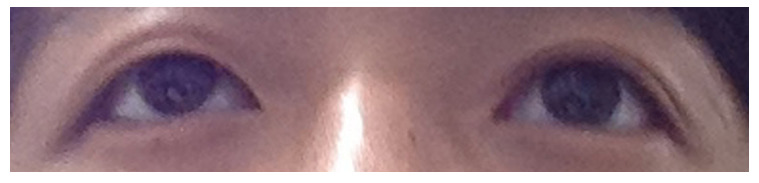

Figure 12: Cut-out eye part

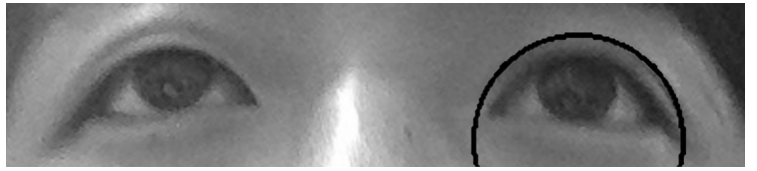

Figure 13: Detection result example 


\section{VERIFICATION EXPERIMENT 2 ON GAZE DETECTION USING EYE DETECTION}

\subsection{Objectives and overview}

Although the results of experiment 1 and the additional experiment showed that the adjustment of contrast would be effective to reduce false detection, they also indicated that eye detection is difficult with circle detection alone. In experiment 2, an OpenCV cascade-type classifier (haarcascade_eye.xml) was installed in the eye detection application for improvement and the success rate of detection was determined. This cascade-type classifier is designed to detect specified objects and was used to detect objects similar to the pre-learned feature values of the eye. When both eyes were detected, detection was considered successful, while only one eye or no eye was detected, it was considered failure. The same 123 photos used in experiment 1 were also used. Considering the results of experiment 1 showing that low contrast would reduce false detection, 3 contrast patterns, -80 , -40, and $0 \%$, were used, and a total of 369 photos (123 multiplied by 3 patterns) were used.

\subsection{Results and consideration}

The detection rate for pattern 1 , the face gazing at the smart phone, was low at about $30 \%$. This is because when gazing at the smart phone screen, the subjects naturally narrowed the eyes, which made the characteristics of the eyes less clear, thus making eye detection difficult. This result indicates that the gazing eyes cannot be used as an eye detection element.

In pattern 2, the straight face looking at the front to see the forward situation, since the subject faces the front, ideally the rate of detection should be as close to $0 \%$ as possible; however, the actual rate was about 15 to $17 \%$. The angle of the smart phone in relation to the face of the subject might have affected. In some cases, even when the subject looked at the front, the eyes were detected.

Meanwhile, the rate of detection for pattern 3 , the downward face with only the eyes looking at the front to see the forward situation, was approximately $60 \%$. When the subject directed the eyes to the front, the eyes became open, which make it easier to capture the eye characteristics, resulting in such a high rate. Both eyes were detected, but at the same time, some false detection occurred on the background. By detecting the face area and focusing only on that area for eye detection, we were able to reduce the number of false detections. The differences in the rate of detection among patterns 1, 2 and 3 were caused by differences in the characteristics and shape

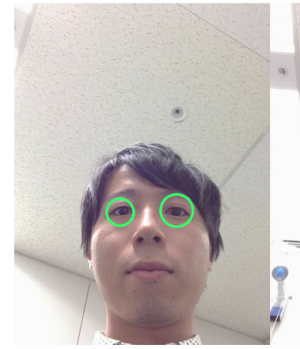

Pattern 1

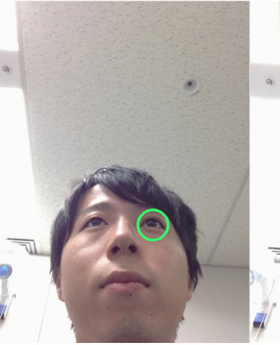

Pattern 2

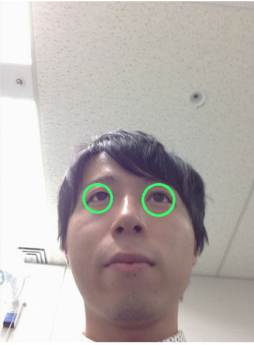

Pattern 3
Figure 14: Eye detection result examples

Table 4: Eye detection rate

\begin{tabular}{|c|c|c|c|}
\hline \multicolumn{4}{|c|}{ Detection rate of contrast } \\
\hline & $-80 \%$ & $-40 \%$ & $0 \%$ \\
\hline Pattern 1 & $\begin{array}{r}26.829 \% \\
(11 / 41)\end{array}$ & $\begin{array}{r}31.707 \% \\
(13 / 41)\end{array}$ & $\begin{array}{r}29.268 \% \\
(12 / 41)\end{array}$ \\
\hline Pattern 2 & $\begin{array}{r}17.073 \% \\
(7 / 41)\end{array}$ & $\begin{array}{r}14.634 \% \\
(6 / 41)\end{array}$ & $\begin{array}{r}17.073 \% \\
(7 / 41)\end{array}$ \\
\hline Pattern 3 & $\begin{array}{r}63.415 \% \\
(26 / 41)\end{array}$ & $\begin{array}{r}58.537 \% \\
(24 / 41)\end{array}$ & $\begin{array}{r}60.976 \% \\
(25 / 41)\end{array}$ \\
\hline
\end{tabular}

of the eyes. We believe that we will be achieve a high detection rate by developing a cascade-type classifier, making the classifier learn different eye characteristics and shapes, and comparing these differences to determine whether or not the smart phone user is gazing at the screen (Figure 14, Table 4).

\section{UTLIZING THE PROPOSED GAZE DETEC- TION SYSTEM}

Numerous studies, including this one, have revealed that the use of smartphones impairs users' consideration of others, as well as their attention to their surroundings, due to their focused gaze on their phone and reduced awareness of their environment. The more focused the users are on their phones, the more dangerous the smartphone use becomes, and the more likely it will lead to accidents. Therefore, it is necessary to design a program that can prevent users from gazing at their mobile devices, by using the gaze detection system developed in this study, in conjunction with other applications. Our system achieved a high accuracy rate of $95.8 \%$ in detecting screen gaze and non-gaze under walking conditions in a verification experiment, and thus is applicable to various walking scenarios. Rather than attempting to prevent users from using their smartphones altogether, the proposed system is best suited to the design of a more useful system that displays the most appropriate message for a given situation, such as "Watch your step, you may cause an accident!" or "Look 


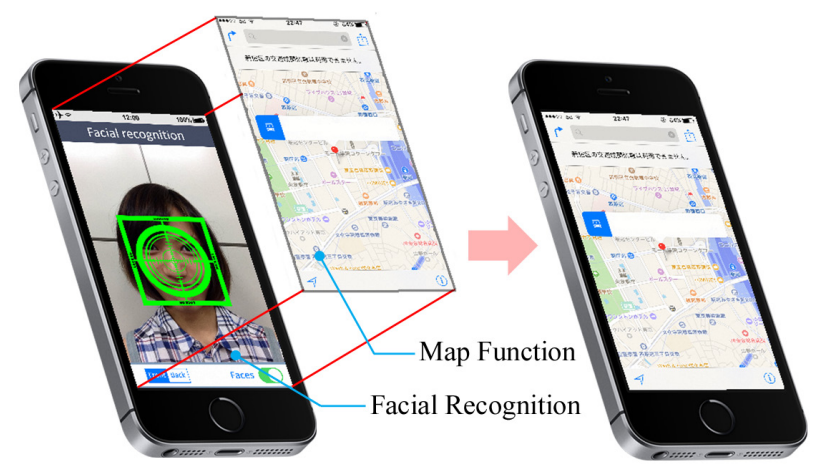

Figure 15: Implementation of the navigation application

up and then proceed!", by measuring the intensity of the user's screen gaze, using the proposed gaze recognition technology, when users are using a navigation (map) application, for example (Figure 15). We are currently in the process of developing a navigation application program that discourages texting while talking, and have been conducting experiments and analysis to verify the system's effectiveness.

\section{CONCLUSIONS AND FUTURE ISSUES}

The questionnaire survey in this study revealed that few people were aware of the dangers of texting while walking although it has becoming a social issue. Therefore, regulations or a highly accurate application to restrict this behavior is required. This study applied facial recognition technology in developing an application program to address the widespread problem of texting while walking. We conducted a verification experiment to assess the application's accuracy in determining facial angles, and the results showed an insignificant difference between the range within which a face could be recognized by the smartphone's built-in camera, and the user's field of vision. Also, the maximum distance for identification fell within a normal range of phone use. These findings, along with the $95.8 \%$ accuracy of the application, confirm the effectiveness of our application in determining whether users are gazing at their smartphone screens, by recognizing their facial angles in real time. In addition, we developed a trial application that detects eye position, after limiting the detection range to the region around the user's face. Though we managed to reduce the false positives by lowering the contrast in captured images during the verification experiment, the application showed low accuracy in detecting eye position; thus, the detection rate must be improved by having a cascade classifier incorporate large amounts of image data. A gaze detection system based on these findings will be able to be used in

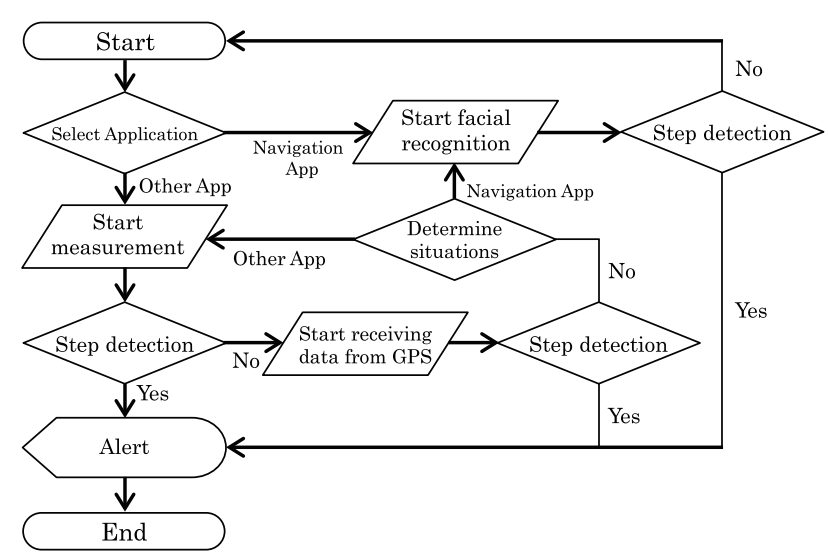

Figure 16: Algorithm used to develop the application to prevent texting while walking

scenarios and user-movement conditions where existing applications are not able to operate, and to detect gaze direction with high accuracy (Figure 16). By embedding the proposed system as a supplementary element in an accelerometer-based application to prevent texting while walking (thus achieving highly accurate step detection), we will be able to discourage users from engaging in the dangerous behavior of texting while walking, and thereby help them break this bad habit, and enable a safer user experience.

We will need to make improvements mainly in the following five areas: (1) illuminance (automatic contrast adjustment), (2) battery depletion, which was pointed out in the questionnaire conducted after experiment 4, (3) incorporation to acceleration-based systems, (4) warning message indication based on the number of face recognitions, etc., and (5) learning by the cascade-type classifier. Also, "Texting While Walking" is because it is a social problem, it thinks that the verification experiment of all validation experiments in this study with respect to expanding the age group not only students are also required.

\section{ACKNOWLEDGEMENTS}

This work was supported by Grant-in-Aid for Scientific Research of Japan.

\section{REFERENCES}

1. au - KDDI; Texting While Walking Attention App, http://www.au.kddi.com/mobile/service/smartphone/ safety/aruki-sumaho/. (in Japanese)

2. Bonsor, K.; How Facial Recognition Systems Work, 2008.

3. Canadian passport photos - Photos - Passport Canada, http://www.ppt.gc.ca/cdn/photos.aspx?lang=eng. 
4. NTT docomo; Texting While Walking Prevention Function, https://www.nttdocomo.co.jp/info/news_release/ 2013/12/03_00.html. (in Japanese)

5. J. Kovač, P. Peer, F. Solina; Human skin color clustering for face detection, EUROCON 2003, Ljubljana, Slovenia, 2003.

6. Kantar Japan; Japanese iPhone share 69.1\%, DOI=http://kantar.jp/whatsnew/2014/01/kantarjapan_ pr_0115.html. (in Japanese)

7. Kohei Nasaka, Takehisa Kato, Masakatsu Nishigaki; A System to Avoid Accidents While Careless Use of Smartphone, IPSJ SIG Technical Report, 2012-DPS150(28), pp.1-6, 2012. (in Japanese)

8. Nikkei BP Consulting; Mobile phones and smartphones "personal use" Survey 2014, 2015. http://consult.nikkeibp.co.jp/consult/news/2014/0829sp/. (in Japanese)

9. NTT docomo; Everyone walked smartphone in Shibuya scramble intersection, 2014. https://www.youtube.com/watch?v=3NDuWV9UAvs. (in Japanese)

10. Ohneda Tomoya, Kamakura Toshinari, Kato Toshikazu; Extraction of focal points in visual searching, The Institute of Image Information and Television Engineers (ITE Technical Report), 33(20), pp.9-12, 2009. (in Japanese)

11. Sanjay Kr. Singh, D.S. Chauhan, Mayank Vatsa, Richa Singh; A Robust Skin Color Based Face Detection Algorithm, Tamkang Journal of Science and Engineering, 6(4), pp.227-234, 2003.

12. Softbank; STOP Texting While Walking, http://www.softbank.jp/corp/group/sbm/news/ press/2014/20140523_01/. (in Japanese)

13. Takumi Negishi, Jiro Tanaka, Tomonari Kamba; Development of “Texting While Walking” Prevention System, Information Processing Society 76th Annual Meeting, 4W-5, pp.301-302, 2014. (in Japanese)

14. Tokyo Fire Department; Note the accident according to walk smartphone !!, 2014.

http://www.tfd.metro.tokyo.jp/lfe/topics/201403/ mobile.html. (in Japanese)

15. Mark Williams; Better Face-Recognition Software, MIT Technology Review, 2008.
16. Yukihiro Okamoto, Kaio Sumi, Kyosuke Makita; An Examination of a Detection Method for Simultaneous Smartphone Operation, DICOM2013, pp.1483-1486, 2013. (in Japanese)

17. Yusuke Abiko, Hidetoshi Onaka; Survey on Generation Tendency and Consciousness of "Texting While Walking", Mobile Society Research Institute, Symposium on Mobile Interactions, pp.59-64, 2014. (in Japanese)

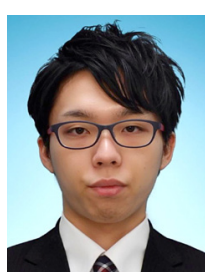

Kento SASAO (Non-member)

Kento Sasao received Bachelor of Informatics from Faculty of informatics, Kogakuin University, Japan, in 2015. In April of the same year, Admission to Kogakuin University Graduate School of Engineering Graduate School of Informatics. Currently a graduate student at Kogakuin University Graduate School. His research interests include Interaction Design, Application Development and Development of a System to Discourage Texting while Walking.

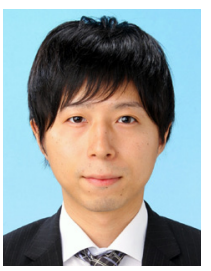

Kazuki OGI (Non-member)

Kazuki Ogi received Bachelor of Informatics from Faculty of informatics, Kogakuin University, Japan, in 2015. Currently, he is a system engineer. His research interests include Interaction Design, Application Development and System Development.

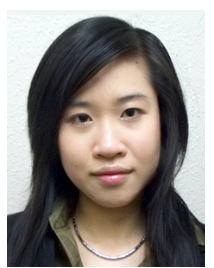

Jue ZHANG (Member)

Jue Zhang is an Associate Professor of Faculty of informatics, Kogakuin University, Japan. She received B.E. degree from Zhejiang University, China. Then received the Master of Design, and $\mathrm{Ph} . \mathrm{D}$. in Kansei Science from Tsukuba University, Japan, in 2004 and 2007, respectively. Her research interests include Information Design, Internet Contents and Kansei Applications. Member of JSKE (Japan), JSSD (Japan).

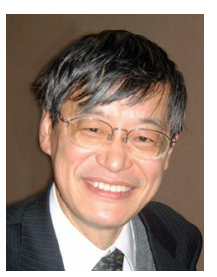

Noboru SUGAMURA (Non-member)

Noboru Sugamura is a Professor of Faculty of informatics, Kogakuin University, Japan. He received Faculty of Engineering Electoronics from Osaka University, Japan. Then received the Master of Ph.D. in Engineering from Osaka University, Japan, in 1976, respectively. He research interests include Foundation and application of Speech Information Processing. Member of IEEE, ACM, IEICE, IPSJ, ASJ, HIS. 